\title{
CORRELAÇÃO ENTRE VOZ, AUTOAVALIAÇÃO VOCAL E QUALIDADE DE VIDA EM VOZ DE PROFESSORAS
}

\author{
Cross-sectional study on voice self-assessment, \\ and quality of life in voice of female teachers
}

\author{
Edna Pereira Gomes de Morais ${ }^{(1)}$, Renata Rangel Azevedo ${ }^{(2)}$, Brasilia Maria Chiari ${ }^{(3)}$
}

\section{RESUMO}

Objetivo: avaliar a qualidade vocal, autoavaliação e a qualidade de vida em voz em professoras do ensino fundamental do $1^{\circ}$ ao $5^{\circ}$ ano e correlacionar os achados. Método: estudo transversal observacional de correlação, com 73 professoras do ensino fundamental do $1^{\circ}$ ao $5^{\circ}$ ano de escolas públicas da Rede Estadual de Educação de Maceió/AL. Aplicou-se um questionário inicial no qual continha uma pergunta de autoavaliação, posteriormente as vozes foram gravadas para análise perceptivo-auditiva por meio da escala analógico-visual e por fim aplicado o questionário de qualidade de vida em voz (QVV). A estatística descritiva foi utilizada para descrever a amostra. O coeficiente de correlação de Spearman foi utilizado para verificar a correlação entre avaliação perceptivo-auditiva, autoavaliação e escores do QVV. Resultados: as professoras apresentaram desvio do comportamento vocal de grau moderado e leve-moderado, respectivamente para emissão sustentada e fala encadeada. A maioria das professoras considerou suas vozes como boas $(37 \%)$ e razoáveis $(43,8 \%)$. O escore total do QVV mostrou impacto médio da voz na qualidade de vida e, apenas a questão de autoavaliação vocal apresentou correlação com os escores do QVV. Os domínios do QVV mostraram correlações significantes entre si. Conclusões: a população estudada apresentou desvio do comportamento vocal e impacto da voz na qualidade de vida, a autoavaliação mostrou satisfação das mesmas com suas vozes, sendo esse aspecto o único a apresentar correlação com os escores do QVV.

DESCRITORES: Saúde Pública; Voz; Docentes; Saúde Ocupacional; Qualidade de Vida

\section{INTRODUÇÃO}

A voz, importante forma de comunicação verbal, é para o professor uma ferramenta indispensável para o exercício de sua profissão, uma vez que institui uma relação dialógica entre o emissor

(1) Fonoaudióloga; Professora Auxiliar do Curso de Fonoaudiologia da Universidade Estadual de Ciências da Saúde de Alagoas - UNCISAL, Maceió, AL, Brasil; Especialização em Voz e em Motricidade Orofacial.

(2) Fonoaudióloga; Professora Adjunta do Departamento de Fonoaudiologia da Universidade Federal de São Paulo UNIFESP, São Paulo, SP, Brasil; Doutora em Distúrbios da Comunicação Humana (Fonoaudiologia) pela Universidade Federal de São Paulo.

(3) Fonoaudióloga; Professora Titular do Departamento de Fonoaudiologia da Universidade Federal de São Paulo UNIFESP, São Paulo, SP, Brasil; Doutora em Distúrbios da Comunicação Humana (Fonoaudiologia) pela Universidade Federal de São Paulo.

Conflito de interesses: inexistente (professor) e o receptor (aluno). Nesse trajeto, emissão-recepção, a presença de alterações vocais podem levar à impedimentos e/ou comprometimentos na atividade docente ${ }^{1,2}$.

No entanto, nem todos os falantes que apresentam voz rouca consideram suas vozes como indicativo de algum distúrbio na laringe e, alguns professores costumam acreditar que as alterações vocais são inerentes à atividade docente, não atentando para os primeiros sintomas de alterações vocais. A falta de uma auto-percepção acurada e conhecimentos sobre a própria voz são apontados por alguns estudos como uma característica comum entre os professores ${ }^{3,4}$. A voz passa a ser um problema quando causa um desconforto e interfere de alguma forma, nas atividades de vida diária. Os professores costumam ser inseridos nos chamados grupos de risco para os distúrbios da voz e, muitas vezes, não procuram tratamento por 
temerem o reconhecimento formal do distúrbio e ficarem rotulados como vulneráveis ${ }^{1,5-7}$.

Qualquer alteração no comportamento vocal do professor não somente produz impacto sobre o próprio sujeito, mas também afeta sua qualidade de vida o que leva à prejuízos tanto no âmbito social quanto profissional ${ }^{8,9}$. A fim de verificar o impacto de uma alteração vocal na qualidade de vida, alguns instrumentos foram desenvolvidos. No Brasil existem atualmente três importantes instrumentos devidamente traduzidos, adaptados e validados, são eles: o questionário de Qualidade de Vida em Voz (QVV), o Perfil de Participação e Atividades Vocais (PPAV) e o Índice de Desvantagem Vocal (IDV). O QVV é considerado um protocolo de autoavaliação e de simples e rápida aplicação; já o PPAV, pode ser usado para mapear áreas de maior impacto de uma disfonia; e o IDV busca a análise da desvantagem que um indivíduo disfônico sofre ${ }^{10-12}$.

As medidas acústicas e perceptivo-auditivas, por si só, não são suficientes para identificar o quanto um problema de voz pode impactar a qualidade de vida das pessoas ${ }^{10,13}$, o que torna necessário acrescentar aos protocolos de avaliação vocal um questionário específico para tal fim, como no caso do protocolo de qualidade de vida em voz.

Apesar dos diversos trabalhos existentes abordando a temática da voz do professor, verifica-se ainda a complexidade do tema e o quanto ainda se faz necessário conhecer sobre o assunto. Correlacionar dados da avaliação perceptivo-auditiva, autoavaliação vocal, e qualidade de vida, permitem um estudo mais amplo da problemática vocal do professor. Assim, o presente estudo teve como objetivo avaliar a qualidade vocal e a qualidade de vida em voz, descrever a autoavaliação vocal e correlacionar os achados em professoras do ensino fundamental do $1^{\circ}$ ao $5^{\circ}$ ano.

\section{MÉTODO}

Estudo transversal observacional de correlação do qual participaram 73 professoras do ensino fundamental do $1^{\circ}$ ao $5^{\circ}$ ano, da Rede Pública Estadual de Educação do Estado de Alagoas, entre maio de 2008 a março de 2009. Como critérios de inclusão foram considerados professores de ambos os sexos, com idade entre 20 e 60 anos e presença de uma ou mais queixa vocal identificada por meio de questionário. Foram excluídos da pesquisa: professores de Educação Física, por terem uma dinâmica profissional diferenciada; professores com menos de um ano de docência; e os que estavam realizando ou realizaram tratamento fonoaudiológico por problemas vocais.
As participantes da pesquisa receberam orientações quanto aos procedimentos da pesquisa. Foi aplicado inicialmente um questionário composto por questões relativas às características sociodemográficas, aspectos relacionados à atividade docente, queixas vocais e autoavaliação vocal. Em seguida, para compor a amostra das vozes que seria avaliada por meio da escala analógico-visual, foi solicitado a cada participante da pesquisa a emissão sustentada da vogal /a/ e fala encadeada (contagem de números de um a dez). Todos os registros foram feitos pela mesma avaliadora, seguindo rigidamente o protocolo de gravação. As vozes foram captadas por meio de microfone unidirecional da marca Gold Ship com condenser, modelo com pedestal de mesa e conector P2, a uma distância de $10 \mathrm{~cm}$ da boca do falante, com ângulo de captação de $45^{\circ}$, acoplado a um notebook da marca Compaq Presario, modelo CQ50-111br, processador Intel Celeron ${ }^{2} 575,2,00$ $\mathrm{GHz}, 1 \mathrm{~GB}$ de RAM, com placa de som da marca SoundMAX Integrated Digital Audio.

Procurou-se manter uma distância do microfone em relação ao notebook, para que não fosse captado o ruído do equipamento. As vozes gravadas foram editadas no programa Sound Forge versão 9.0, desprezando-se os três segundos iniciais de cada emissão da vogal e consequentemente, o ataque vocal.

$\mathrm{Na}$ edição das vozes, para se verificar a confiabilidade entre os avaliadores, foi gerado um arquivo no qual foram armazenadas todas as amostras de vozes, acrescidas de $10 \%$ de repetição aleatória, totalizando assim 81 registros, que ficaram armazenadas em CD com extensão mp3, para posterior análise perceptivo-auditiva.

Posteriormente, as vozes gravadas foram avaliadas por três fonoaudiólogos especialistas em voz, com tempo de atuação superior a cinco anos na clínica de voz. Os profissionais avaliaram o desvio do comportamento vocal por meio da análise perceptivo-auditiva, utilizando-se a escala analógico-visual ${ }^{14,15}$.

Os dados foram resumidos por meio da frequência para cada uma das faixas de distribuição dos graus de desvio do comportamento vocal em pontos, considerando-se variabilidade normal o desvio compreendido entre 0 e $35,5 \mathrm{~mm}$; leve a moderado, 35,6 a 50,5mm; moderado, 50,6 a $90,5 \mathrm{~mm}$ e intenso, 90,6 a 100 mm, conforme critérios estabelecidos na literatura ${ }^{15}$.

A presente pesquisa foi aprovada pelo Comitê de Ética em Pesquisa da Universidade Estadual de Ciências da Saúde de Alagoas - UNCISAL, sob o parecer de no 803/07 e pelo Comitê de Ética em Pesquisa da Universidade Federal de São Paulo UNIFESP, sob o parecer de no 0761/09, de acordo 
com a Resolução 196/96, da Comissão Nacional de Ética em Pesquisa - CONEP, que versa sobre as diretrizes e normas regulamentadoras de pesquisas envolvendo seres humanos. Todos os sujeitos envolvidos na pesquisa assinaram o Termo de Consentimento Livre e Esclarecido, concordando em participar do estudo e com a divulgação dos resultados.

Para avaliação da qualidade de vida em voz foi aplicado o Protocolo de Qualidade de Vida em Voz (QVV), traduzido e adaptado do Voice Related Quality of Life (V-RQOL) ${ }^{16}$ e recentemente validado ${ }^{11}$. O mesmo consiste em um questionário de autoavaliação vocal que busca investigar o impacto da voz na qualidade de vida, sendo um questionário composto por dez questões, as quais se dividem em dois domínios: socioemocional (questões de número 4, 5, 8 e 10) e físico (questões de número 1, $2,3,6,7$ e 9), que testam dois aspectos relevantes e diferentes de como um distúrbio vocal pode interferir no cotidiano do indivíduo ${ }^{11,16}$. O questionário produz três diferentes escores - físico, socioemocional e total, e os resultados podem variar de 0 a 100 , em que 0 indica uma qualidade de vida baixa; e 100, excelente; os escores de 81 a 100 indicam baixo impacto da voz na qualidade de vida; de 61 a 80 médio impacto; e menor ou igual a 60 , alto impacto da voz na qualidade de vida ${ }^{17}$.

A estatística descritiva, por meio da média, d.p.(desvio padrão), valor mínimo e máximo e mediana, foi utilizada para descrever a amostra do presente estudo.

Após a identificação do avaliador de maior confiabilidade, por meio do cálculo do coeficiente de correlação intraclasse $(\mathrm{CCl})$, foi construída uma única tabela com os resultados referentes à emissão da vogal sustentada /a/ e fala encadeada, a qual foi utilizada para a correlação com as demais variáveis.

Para comparar as médias obtidas por cada avaliador na avaliação perceptivo-auditiva, foi utilizada a análise de variância (ANOVA), por se tratar de variáveis com distribuição contínua (escala analógico-visual).

As correlações entre os escores do QVV, a questão de autoavaliação "como você avalia sua voz?" e avaliação perceptivo-auditiva por meio da escala analógico-visual para a emissão sustentada da vogal /a/ e fala encadeada (contagem de um a dez) foram feitas por meio do coeficiente de correlação de Spearman.

O grau de correlação entre as variáveis foi considerado maior quanto mais próximo de -1 ou de 1 , sendo as correlações negativas ou positivas, respectivamente.

O teste de Mann-Whitney foi utilizado para comparar as médias dos resultados da avaliação perceptivo-auditiva para a vogal sustentada /a/ e fala encadeada e as médias dos domínios socioemocional e físico do QVV, considerado significante para $\mathrm{p}<0,05$.

Todos os cálculos estatísticos foram efetuados por meio do software estatístico Minitab, versão 15.1.

\section{RESULTADOS}

A média de idade para a amostra estudada foi de 43,6 \pm 6,7 anos com variação de 25 a 55 anos. $O$ tempo de magistério foi de 2 a 29 anos, com média de 14,9 $\pm 7,8$ anos. A carga horária semanal de trabalho apresentou variação de 6 a 60 horas/semanais, com média de 36,9 \pm 9,8 horas/semanais.

A tabela 1 apresenta a frequência de respostas e percentagem das variáveis idade, tempo de magistério, carga horária, queixas vocais mais referidas e respostas à questão de autoavaliação "como você avalia sua voz?"

$\mathrm{Na}$ tabela 2 estão descritos, por meio das medidas de tendência central, os resultados da avaliação perceptivo-auditiva por meio da escala analógico-visual (EAV) para a vogal /a/ sustentada e fala encadeada (contagem de um a dez), domínios e escore total do QVV e teste de Mann-Whitney para as médias da avaliação perceptivo-auditiva e domínios socioemocional e físico do QVV.

A tabela 3 apresenta o Coeficiente de correlação de Spearman para o cruzamento entre os domínios e escore total do QVV com a questão "como você avalia sua voz?", avaliação perceptivo-auditiva por meio da escala analógico-visual (EAV) para vogal sustentada /a/ e fala encadeada e entre os domínios do QVV. 
Tabela 1 - Descrição da frequências de respostas e percentagem das variáveis idade, tempo de magistério, carga horária, queixas vocais mais referidas e respostas à questão de autoavaliação "como você avalia sua voz?"

\begin{tabular}{|c|c|c|}
\hline Variável & $\mathbf{N}$ & $\%$ \\
\hline \multicolumn{3}{|l|}{ Idade (anos) } \\
\hline $20-130$ & 01 & 1,4 \\
\hline $30-40$ & 19 & 26,0 \\
\hline $40-50$ & 41 & 56,2 \\
\hline $50-60$ & 12 & 16,4 \\
\hline Total & 73 & 100 \\
\hline \multicolumn{3}{|l|}{ Tempo de magistério (anos) } \\
\hline $01-05$ & 07 & 9,6 \\
\hline $05-10$ & 28 & 38,3 \\
\hline $10-15$ & 02 & 2,8 \\
\hline $15-20$ & 13 & 17,8 \\
\hline $20-25$ & 20 & 27,4 \\
\hline $25-30$ & 03 & 4,1 \\
\hline Total & 73 & 100 \\
\hline \multicolumn{3}{|l|}{ Carga horária semanal (horas) } \\
\hline $0-\mid 20$ & 12 & 16,5 \\
\hline $20-130$ & 04 & 5,4 \\
\hline $30-40$ & 51 & 69,9 \\
\hline $40-50$ & 03 & 4,1 \\
\hline $50-60$ & 03 & 4,1 \\
\hline Total & 73 & 100 \\
\hline \multicolumn{3}{|l|}{ Queixas vocais mais referidas } \\
\hline Rouquidão & 67 & 91,8 \\
\hline Cansaço ao falar & 43 & 58,9 \\
\hline Quebras na voz & 31 & 42,5 \\
\hline Garganta seca & 15 & 20,5 \\
\hline Dor/ardor ao falar & 14 & 19,2 \\
\hline \multicolumn{3}{|l|}{ "Como você avalia sua voz?" } \\
\hline Excelente & 01 & 1,4 \\
\hline Muito boa & 04 & 5,5 \\
\hline Boa & 27 & 37,0 \\
\hline Razoável & 32 & 43,8 \\
\hline Ruim & 09 & 12,3 \\
\hline Total & 73 & 100 \\
\hline
\end{tabular}

Legenda: $\mathrm{N}$ = número de frequência de respostas; \% = frequência em percentagem 
Tabela 2 - Resultados da avaliação perceptivo-auditiva por meio da escala analógico-visual para a vogal /a/ sustentada e fala encadeada (contagem de um a dez), domínios e escore total do QVV

\begin{tabular}{lccccccc}
\hline & N & Média & d.p. & Mínimo & Mediana & Máximo & P \\
\hline EAV & 73 & 57,2 & 15,1 & 22 & 61 & 91 & \\
$\quad$ vogal sustentada & 73 & 50,4 & 10,9 & 28 & 50,5 & 72 & $<0,001$ \\
$\quad$ fala encadeada & & & & & & & \\
QVV & 73 & 80,5 & 26,4 & 0 & 94 & 100 & $<0,001$ \\
$\quad$ Socioemocional & 73 & 66,2 & 24,2 & 13 & 67 & 100 & \\
$\quad$ Físico & 73 & 72,0 & 23,6 & 10 & 78 & 100 & \\
$\quad$ Total &
\end{tabular}

Legenda: $\mathrm{EAV}$ = escala analógico-visual; $\mathrm{QVV}=$ qualidade de vida em voz; $\mathrm{N}=$ número de professoras avaliadas; d.p. = desvio padrão; $p$ = nível de significância do teste Mann-Whitney. Nível de significância adotado de $p<0,05$.

Tabela 3 - Coeficiente de correlação de Spearman para o cruzamento entre os domínios e escore total do QVV com a questão "como você avalia sua voz?", avaliação perceptivo-auditiva por meio da EAV para vogal sustentada /A/ e fala encadeada e entre os domínios do QVV

\begin{tabular}{lcccccc}
\hline & \multicolumn{2}{c}{ Socioemocional } & \multicolumn{2}{c}{ Físico } & \multicolumn{2}{c}{ Escore Total } \\
\cline { 2 - 7 } & c & p-valor & C & p-valor & c & p-valor \\
\hline Socioemocional & - & - & 0,802 & $0,000^{*}$ & 0,894 & $0,000^{*}$ \\
Físico & 0,802 & $0,000^{*}$ & - & - & 0,977 & $0,000^{*}$ \\
Escore Total & 0,894 & $0,000^{*}$ & 0,977 & $0,000^{*}$ & - & - \\
Como você avalia sua voz? & $-0,371$ & $0,001^{*}$ & $-0,531$ & $0,000^{*}$ & $-0,504$ & $0,000^{*}$ \\
EAV-vogal /a/ sustentada & $-0,670$ & 0,572 & $-0,102$ & 0,390 & $-0,086$ & 0,467 \\
EAV-fala encadeada & $-0,037$ & 0,758 & $-0,161$ & 0,174 & $-0,116$ & 0,327 \\
\hline
\end{tabular}

Legenda: Correlação de Spearman; $c=$ correlação; $p$-valor = valor de $p$, significante para $p \leq 0,05 ;{ }^{*}=$ Valor de $p$ quando significante; $\mathrm{EAV}=$ escala analógico-visual; EAV-vogal /a/ sustentada = escala analógico-visual para vogal sustentada /a/; EAV-fala encadeada= escala analógico-visual para a fala encadeada (contagem de 1 a 10).

\section{DISCUSSÃO}

Os estudos acerca da voz do professor apontam o sexo feminino como predominante na educação brasileira, principalmente no que diz respeito ao ensino infantil e fundamental de $1^{\circ}$ ao $5^{\circ}$ ano ${ }^{18-20}$. As mulheres são apontadas como mais predispostas à problemas vocais que os homens, principalmente se essa apresenta carga horária vocal elevada para o exercício de sua atividade profissional, como no caso das professoras ${ }^{21}$. Pode-se ainda relacionar os vários papéis sociais assumidos pelas mulheres, das responsabilidades familiares e trabalhos domésticos que têm que assumir, o que pode configurar uma dupla jornada de trabalho ${ }^{21,22}$.

De acordo com a literatura, a variável idade merece atenção, pois à medida que a idade avança, a eficiência vocal diminui; a faixa etária entre 25 e 45 anos é, então, considerada a de maior eficiência vocal e, a partir desse período, uma série de alterações estruturais na laringe podem ocorrer, com maior ou menor impacto vocal ${ }^{23}$. Na população estudada a média de idade está em 43,6 anos, com maior concentração das professoras entre a faixa etária de 31 a 50 anos (60; 82,2\%).

Os dados encontrados no estudo em questão quanto a carga horária semanal média, apontaram maior frequência para carga horária semanal de 21 a 40 horas $(55 ; 75,3 \%)$, resultados que assemeIham-se aos achados de outros estudos ${ }^{21,24}$.

Ao considerar a classe mais frequente, na tabela 1, quanto ao tempo de magistério, pode-se afirmar que a maioria das professoras são jovens quanto ao tempo de atuação profissional, entretanto 23 $(31,5 \%)$ professoras pesquisadas faziam uso profissional da voz há mais de 20 anos. Os professores em início de carreira necessitam de atenção tanto quanto aqueles com mais anos de atuação profissional, no que diz respeito a saúde vocal. Os cuidados com a voz do professor deveriam iniciar-se nos cursos de graduação, com programas de prevenção e informações acerca do uso voz $z^{5,21,23,25}$.

De acordo com alguns pesquisadores as alterações vocais em professores são multifatoriais ${ }^{18,26,27}$. 
O ambiente de trabalho impróprio, o número excessivo de alunos em sala de aula, que também obriga o professor a aumentar a intensidade da voz para ser ouvido por todos na sala, jornada elevada de trabalho, uso inadequado da voz e falta de organização do trabalho, além da indisciplina dos alunos, que gera desgaste adicional para o profissional, colocam os professores em grupo de risco para os distúrbios vocais ${ }^{1,7}$. No presente estudo pode-se observar a presença de alguns desses fatores como a carga horária excessiva e o tempo de magistério, que se enquadram, então, como fatores que colaboram para o surgimento de alterações vocais, que podem levar a futuros afastamentos e até readaptações ao trabalho. Tais achados podem ter contribuído para a presença das queixas vocais autorreferidas pelo grupo estudado.

A sobrecarga à qual o professor se submete é um fator importante a ser considerado na etiologia dos desvios vocais. Os baixos salários são, muitas vezes, o impulso para a elevada carga horária, o que pode vir a comprometer a qualidade de vida. As alterações vocais em professores merecem atenção especial pelo papel que a voz de tais profissionais desempenha em sala de aula como instrumento de comunicação e informação ${ }^{22}$.

Os achados quanto à sintomatologia vocal encontrados e demonstrados na tabela 1, corroboram com os resultados de outros estudos ${ }^{28,29}$, os quais mostraram que entre os sintomas vocais mais relatados pelos professores estão rouquidão, fadiga vocal, voz estridente, ardor e/ou dor na região da garganta e pescoço, dificuldades em manter a voz, variações na frequência fundamental, falta de volume e projeção vocal, perda da eficiência vocal, pouca resistência ao falar e afonia ou perda total da voz. A rouquidão foi o sintoma mais referido pela população estudada, e apresenta-se como a queixa comum entre a maioria dos indivíduos que exercem atividade docente.

Estudos anteriores revelaram achados semeIhantes aos aqui apresentados quanto a média de idade, carga horária semanal e tempo de profissão ${ }^{13,29}$.

Os resultados da tabela 1 evidenciam que mesmo havendo uma grande referência à rouquidão, as professoras encontram-se satisfeitas com suas vozes, o que corrobora com estudos anteriores ${ }^{4,21}$ e mostra-se contrário a outro ${ }^{2}$, onde os sujeitos que avaliaram suas vozes como alteradas obtiveram essa confirmação por meio de avaliação fonoaudiológica e aquelas que não as julgaram como alteradas, não apresentaram alterações na avaliação feita pelo fonoaudiólogo.

Mesmo com a presença das queixas vocais apresentadas, a voz não foi, todavia, impedimento para o exercício da atividade docente no grupo estudado, uma vez que todas as professoras encontravam-se em sala de aula desenvolvendo suas funções pedagógicas. Os achados quanto a autoavaliação vocal mostram a dificuldade das professoras atentarem para os primeiros sinais de alterações na voz, ou seja, o desconhecimento quanto a própria $v_{0} z^{4}$.

Os resultados da avaliação perceptivo-auditiva por meio da escala analógico-visual (EAV), apresentados na tabela 2, para vogal sustentada /a/ e fala encadeada, mostraram que há presença de desvio do comportamento vocal entre as professoras da pesquisa, ocorrendo com grau de desvio moderado para emissão sustentada da vogal /a/ e leve a moderado para fala encadeada. Tais dados revelam que a população estudada é de professores com disfonia, porém com graus variados de alterações e semelhantes ao de outro estudo ${ }^{5}$.

Os dados aqui encontrados permitem justificar a necessidade de programas que visem o bem estar vocal para essa população, na tentativa de minimizar a ocorrência dos sintomas referidos entre esses profissionais.

Apesar da presença de desvio no comportamento vocal na maioria das professoras da pesquisa, as mesmas encontram-se satisfeitas com suas vozes, o que pode implicar estratégias de enfrentamentos que mascaram suas alterações e permitem o desempenho de suas atividades profissionais, ou até mesmo ao fato de estarem adaptadas às suas vozes, pois mesmo alteradas ainda possuem voz para exercício da atividade laboral, importando "ter voz", como demonstrado por outros estudos².

A literatura aponta a disfonia como uma manifestação comum entre os professores, levando a impactos tanto na esfera profissional como na qualidade de vida ${ }^{5,30}$.

O fato da vogal /a/ ter apresentado um grau de desvio diferente da fala encadeada mostra a influência dos articuladores na produção desta, uma vez que, para falar, os ajustes se modificam em função das estruturas do trato vocal (filtro) que ajudam, muitas vezes, os indivíduos com alterações vocais a mascararem seus comportamentos vocais alterados ${ }^{31}$.

Os resultados do presente estudo, comparados à literatura estudada, reforçam a importância de constar nas avaliações vocais protocolos que busquem e valorizem a autopercepção do indivíduo quanto a sua voz, o que gera uma maior dimensão dessa avaliação. Assim, além de conhecer e traçar o perfil vocal dos professores é preciso dar-lhes instrumentos que os façam refletir sobre sua própria voz e despertar para os primeiros sinais e sintomas de alterações vocais. Uma avaliação vocal deveria 
ser composta, no entanto, por: avaliação perceptivo-auditiva e visual, a avaliação acústica, e protocolos de autoavaliação $0^{8,10}$.

Ao pensar na produção vocal em relação à saúde geral, as alterações vocais podem impactar a qualidade de vida do sujeito. Apesar de existir há algum tempo em outras áreas da saúde, os cuidados com a qualidade de vida em pacientes disfônicos tem sido uma preocupação recente na Fonoaudiologia ${ }^{8}$.

Os dados apresentados na tabela 2, quanto aos resultados do QVV, mostram que as vozes das professoras estudadas apresentaram um impacto na qualidade de vida delas, com valor de 72 pontos para o escore total. A literatura aponta que escores com valores entre 61 e 80 , implicam em um grau de impacto médio da voz na qualidade de vida ${ }^{17}$. Os aspectos físicos, por estarem relacionados ao desconforto e queixas apresentadas para o uso da voz, favorecem uma maior percepção dos sujeitos para julgarem como o mais impactante. Os achados aqui obtidos quanto a qualidade de vida em voz, corroboram com os de pesquisas anteriores ${ }^{18,24}$.

Os resultados do presente estudo mostraram a presença de queixas vocais e desvio do comportamento vocal, este último comprovado por avaliação fonoaudiológica, em uma população que continua em atividade profissional sem acompanhamento e/ ou orientações fonoaudiológica, conforme critérios de inclusão.

A presença de programas de bem estar vocal voltado a essa população permitiriam o desenvolvimento de uma percepção mais acurada de suas vozes, com despertar para o problema vocal aos primeiros sinais/sintomas de alterações, devendo ainda o trabalho não limitar-se a essa esfera, mas também a um aprimoramento da competência comunicativa em sala de aula, bem como permitir assistência a esses sujeitos que, muitas vezes, não têm condições de buscar um atendimento fonoaudiológico que não seja gratuito.

Os professores deveriam ser incentivados à busca de orientações e tratamento, evitando-se uma alteração vocal maior, com impacto negativo do ponto de vista profissional, social e emocional. $O$ professor sem alterações terá mais disposição para o desempenho de sua atividade profissional, mas tem-se que considerar a participação dos gestores nesse processo e a adequação do ambiente de trabalho ${ }^{1,4}$. Verifica-se, por meio de estudos publicados a presença de programas desse tipo em diversas regiões do Brasil, porém ainda escassos na região nordeste ${ }^{28}$, apesar de existirem discussões a respeito.

Ao se estabelecer uma correlação entre os escores socioemocional, físico e total do QVV, observou-se a presença de correlação boa entre o domínio físico e escore total com o domínio socioemocional; para o domínio físico e escore total a correlação foi considerada ótima, conforme mostra a Tabela 3. Tais achados implicam dizer que quanto maior for o escore do domínio socioemocional, maior será o valor do domínio físico e vice-versa, bem como à medida que esses crescem o escore total também aumenta. Esses resultados corroboram com os encontrados em estudo semelhante ${ }^{24}$.

A correlação entre o grau de desvio do comportamento vocal, tanto para vogal sustentada quanto para fala encadeada, não mostrou correlação significante com os escores socioemocional, físico e total do QVV. No entanto, ao correlacionar a questão "como você avalia sua voz?" com os escores socioemocional, físico e total do QVV, pôde-se observar a presença de correlação significativa. Esses resultados apontam que quanto pior for a autoavaliação do indivíduo em relação a sua voz, piores serão os resultados dos domínios e escore total do QVV. Tais resultados assemelham-se aos encontrados por outros estudos acerca da qualidade de vida em VOZ $^{19,21}$.

Diante de todos os resultados encontrados e discutidos, acredita-se que o surgimento de programas de bem estar vocal para professores permitiriam um melhor entendimento do problema vocal, despertando para uma autoavaliação mais aprimorada e minimizando a instalação de um problema vocal que leve às licenças, afastamentos e desvios de função tão comuns aos professores com disfonia.

Seria importante considerar que o desenvolvimento de ações preventivas fossem iniciadas no período de formação docente e, perpetuassem ao longo da carreira. Para o professor a voz é o seu principal meio de comunicação e deve se apresentar de forma saudável, eficiente e sem esforço, ser interessante e clara, a fim de garantir a atenção do aluno'.

\section{CONCLUSÃO}

Pode-se concluir com o presente estudo a presença de desvio do comportamento vocal na população estudada, com graus de alteração diferentes entre uma emissão sustentada e fala encadeada; presença de impacto da alteração vocal na qualidade de vida relacionada à voz; e ausência de correlação entre avaliação perceptivo-auditiva e escores do QVV. Ao contrário, a questão de autoavaliação "como você avalia sua voz" apresentou correlação significante com os escores do QVV, o que implica dizer que quanto pior for a autoavaliação piores serão os escores do QVV. 


\section{ABSTRACT}

Purpose: to evaluate vocal quality and voice-related quality of life in 1st to 5th grade female teachers, to check vocal self-assessment, and to correlate those findings. Method: an observational crosssectional study on the correlation with seventy-three 1 st to 5 th grade teachers in state public elementary schools in the city of Maceió/AL. An objective questionnaire was used in order to characterize the said sample. An auditory perceptual analysis of the voices was conducted and the voice-related quality of life questionnaire (V-RQOL) was administered. The sample was described by using descriptive statistics. The Spearman's rank correlation coefficient was used to assess the correlation among auditory perceptual analysis, self-assessment, and voice-related quality of life scores. Results: teachers suffered from moderate vocal deviation in sustained phonation and mild to moderate deviation in connected speech. Most teachers deemed their voices to be good (37\%) and acceptable (43.8\%). As to vocal complaints, hoarseness was the most cited (91.8\%). Total scores for V-RQOL showed moderate impact of the voice on quality of life, and only the vocal self-assessment issue had any correlation to V-RQOL scores. V-RQOL domains showed significant correlations amongst themselves. Conclusion: the population in the study suffered from vocal deviation and its impact on quality of life, although most of them were pleased with their voices. Nothing, but the vocal assessment topic had any correlation with the V-RQOL scores, implying that the worse is the vocal self-assessment, the worse is the voice-related quality of life scores.

KEYWORDS: Public Health; Voice; Faculty; Occupational Health; Quality of Life

\section{REFERÊNCIAS}

1. Vieira AC, Behlau M. Análise de voz e comunicação oral de professores de curso pré-vestibular. Rev Soc Bras Fonoaudiol. 2009;14(3):346-51.

2. Simões M, Latorre MRDO. Prevalência de alteração vocal em educadoras e sua relação com a auto-percepção. Rev Saúde Pública [Internet]. 2006;40(6):1013-8 Disponível em: http://www. scielosp.org/pdf/rsp/v40n6/08.pdf, DOI:10.1590/ S0034-89102006000700008.

3. Munier C, Kinsella R. The prevalence and impact of voice problems in primary school teachers. Occup Med.2008;58(1):74-6.

4. Fabrício MZ, Kasama ST, Martinez EZ. Qualidade de vida relacionada à voz de professores universitários. Rev CEFAC [online]. 2009; 12(2): 280-7. Disponível em: http://www.scielo. br/pdf/rcefac/v12n2/183-08.pdf, DOI: 10.1590/ S1516-18462009005000062

5. Azevêdo LL, Vianello L, Oliveira HGP, Oliveira IA, Oliveira BFV, Silva CM. Queixas vocais e grau de disfonia em professoras do ensino fundamental. Rev Soc Bras Fonoaudiol [Internet]. 2009;14(2):192-6. Disponível em: http://www. scielo.br/pdf/rsbf/v14n2/09.pdf, DOI:10.1590/ S1516-80342009000200009.

6. Tavares ELM, Martins RHG. Vocal evaluation in teachers with and without symptoms. J Voice. 2007;21(4):407-14, DOI:10.1016/j.jvoice. 2006.04.001.
7. Araújo TM, Reis EJF, Carvalho FM, Porto LA, Reis IC, Andrade JM. Fatores associados a alterações vocais em professoras. Cad Saúde Pública. 2008; 24(6):1229-38.

8. Behlau M, Feijó D, Madázio G, Rehder MI, Azevedo R, Ferreira AE. Voz profissional: aspectos gerais e atuação fonoaudiológica. In: Behlau M. Voz: o livro do especialista. Rio de Janeiro: Revinter; 2005. p. 287-407.

9. Park K, Behlau M. Perda vocal em professores e não professores. Rev Soc Bras Fonoaudiol. 2009;14(3):463-9.

10. Behlau M, Oliveira G, Santos LMA, Ricarte A. Validação no Brasil de protocolos de auto-avaliação do impacto de uma disfonia. Pró-Fono. 2009;21(4):326-32. DOI:10.1590/ S0104-56872009000400011.

11. Gasparini G, Behlau M. Quality of life: validation of the Brazilian version of the voice-related quality of life (V-RQOL) measure. J Voice. 2009;23(1):76-81. DOI:10.1016/j.jvoice.2007.04.005

12. Kasama ST, Brasolotto AG. Percepção vocal e qualidade de vida. Pró-Fono. 2007;19(1):19-28.

13. Ma EP-M, Yiu EM-L. Voice Activity and Participation Profile: Assessing the Impact of Voice Disorders on Daily Activities $J$ Speech Lang Hear Res. 2001;44:511-24, DOI:10.1044/1092-4388(2001/040)

14. Yamasaki R, Leão SHS, Madazio G, Padovani $\mathrm{M}$, Azevedo R. Análise perceptivo-auditiva de vozes normais e alteradas: Escala analógica visual. XV 
Congresso Brasileiro de Fonoaudiologia e VII Congresso Internacional de Fonoaudiologia; 2007; Gramado. São Paulo: Sociedade Brasileira de Fonoaudiologia; 2007.

15. Yamasaki R, Leão SHS, Madazio G, Padovani $M$, Azevedo R, Behlau M. Correspondência entre Escala Analógico-Visual e a Escala Numérica na Avaliação Perceptivo-Auditiva de Vozes. XVI Congresso Brasileiro de Fonoaudiologia; 2008; Campos de Jordão. São Paulo: Sociedade Brasileira de Fonoaudiologia; 2008.

16. Hogikyan ND, Sethuraman G. Validation of an instrument to measure voice-related quality of life (V-RQOL). J Voice. 1999;13(4):557-69.

17. Servilha EAM, Roccon PF. Relação entre voz e qualidade de vida em professores universitários. Rev CEFAC [Internet]. 2009;11(3):440-8. Disponível em: www.scielo.br/pdf/rcefac/2009nahead/42-08. pdf DOI:10.1590/S1516-18462009005000029.

18. Jardim R, Barreto SM, Ávila AA. Condições de trabalho, qualidade de vida e disfonia entre docentes. Cad Saúde Pública [Internet]. 2007a;23(10):2439-61. Disponível em: http://www. scielo.br/pdf/csp/v23n10/19.pdf, DOI:10.1590/ S0102-311X2007001000019.

19. Penteado RZ, Bicudo-Pereira IMT. Avaliação do impacto da voz na qualidade de vida de professores. Rev Soc Bras Fonoaudiol. 2003;8(2):19-28.

20. Troni CR, Arakaki FN, Lima FS, Mott L, Rodrigues KA, Ferreira LP. Professores em contexto profissional e não profissional: análise objetiva e subjetiva dos aspectos da articulação e da postura. Distúrbios da Comunicação. 2006;18(2):179-88.

21. Grillo MH, Penteado RZ. Impacto da voz na qualidade de vida de professore(a)s do ensino fundamental. Pró-Fono. 2005;17:321-30.

22. Laukkanen A-M, Ilomäki I, Leppänen K, Vilkman, E. Acoustic Measures and Selfreports of Vocal Fatigue by Female Teachers. J Voice. 2008;22(3):283-9, DOI:10.1016/j. jvoice.2006.10.001

23. Kooijman PGC, Thomas G, Graamans K, de Jong FICRS. Psychosocial Impact of the Teacher's
Voice Throughout the Career. J Voice. 2005; 21(3):316-24.

24. Jardim R, Barreto SM, Assunção AÁ. Disfonia: definição de caso e prevalência em professores. Rev Bras Epidemiol [online]. 2007b;10(4):625-36. Disponível em: http://www. scielo.br/pdf/rbepid/v10n4/19.pdf DOI:10.1590/ S1415-790X2007000400020.

25. Simberg S, Sala E, Rönnema AM. A comparison of the prevalence of vocal symptoms among teacher students and other university students. J Voice. 2004;18(3):363-8. DOI:10.1016/j. jvoice.2003.12.005

26. Gonçalves VSB, Silva LB, Coutinho AS. Ruído como agente comprometedor da inteligibilidade de fala dos professores. Prod [internet]. 2009;19(3):466-76. Disponível em: http://www. scielo.br/pdf/prod/v19n3/05.pdf DOI: 10.1590/ S0103-65132009000300005.

27. Roy N, Merrill RM, Thibeault S, Parsa RA, Gray SD, Smith EM. Prevalence of voice disorders in teachers and the general population. J Speech Lang Hear Res. 2004;47(2):281-93. DOI: 10.1044/1092-4388(2004/023)

28. Ferreira LP, Servilha EAM, Masson MLV, Reinaldi MBFM. Políticas públicas e voz do professor: caracterização das leis brasileiras. Rev Soc Bras Fonoaudiol [Internet]. 2009;14(1):1-7. Disponível em: www.scielo.br/pdf/rsbf/v14n1/03.pdf DOI:10.1590/S1516-80342009000100003.

29. Silverio KC, Gonçalves CG, Penteado RZ, Vieira TP, Libardi A, Rossi D. Ações em saúde vocal: proposta de melhoria do perfil vocal de professores. Pró-Fono [Internet]. 2008;20(3):177-82. Disponível em: $\quad$ www.scielo.br/pdf/pfono/v20n3/en_07.pdf DOI:10.1590/S0104-56872008000300007.

30. Behlau M, Hogikyan ND, Gasparini G. Quality of life and voice: study of a Brazilian population using the voice-related quality of life measure. Folia Phoniatr Logop. 2007;59(6):286-96.

31. Bele I. Reliability in perceptual analysis of voice quality. J Voice. 2005;19(4):555-7. DOI:10.1016/j. jvoice.2004.08.008

http://dx.doi.org/10.1590/S1516-18462012005000032

RECEBIDO EM: 11/07/2011

ACEITO EM: 10/11/2011

Endereço para correspondência:

Edna Pereira Gomes de Morais

Rua Miguel Palmeira, 1318 - Pinheiro

Maceió - AL

CEP: $57077-330$

E-mail: epgmorais@yahoo.com.br 